\title{
Recurrent prosthetic mitral valve infective endocarditis and perivalvular abscess: first description by PET/CT angiography
}

\author{
Jordi Lozano-Torres ${ }^{1}$ - María Nazarena Pizzi ${ }^{1}$ Albert Roque ${ }^{1}$.

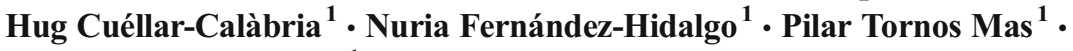 \\ Santiago Aguadé-Bruix ${ }^{1}$
}

Received: 8 April 2016 / Accepted: 28 April 2016/Published online: 13 May 2016

(C) Springer-Verlag Berlin Heidelberg 2016

Keywords Prosthetic valve · Infective endocarditis .

Perivalvular abscess $\cdot$ PET/CT

A 69-year-old woman with a history of rheumatic valvulopathy requiring mechanical prosthetic valve substitution 11 years previously was admitted to our service because of fever. Physical exploration was unremarkable. Blood cultures were positive for cloxacillin-resistant Staphylococcus epidermidis. The echocardiogram showed a large mitral vegetation without signs of perivalvular abscess or prosthetic dysfunction. The patient received a 6-week regimen of daptomicin, gentamicin and rifampicin, with immediate negativization of the blood cultures and a good evolution. However, blood cultures became positive for the same microorganism 2 weeks after the end of the treatment. Repeat transoesophageal echocardiography showed only a small residual vibrating mass.

${ }^{18}$ F-FDG PET/CT angiography with myocardial suppression showed a periprosthetic mitral abscess that was able to explain the recurrent endocarditis. The oblique coronal view (a) shows a large residual nodular calcification of the posterior mitral ring. Short-axis, two-chamber and four-chamber fused images in the mitral plane $(b-d)$ show intense focal FDG uptake (SUVmax 13) in the same location [1,2].

The patient underwent surgery during which a periprosthetic mitral abscess at the inferior ring was completely

Jordi Lozano-Torres

jordilozano87@gmail.com

1 Hospital Universitari Vall d'Hebron, Barcelona, Catalunya, Spain

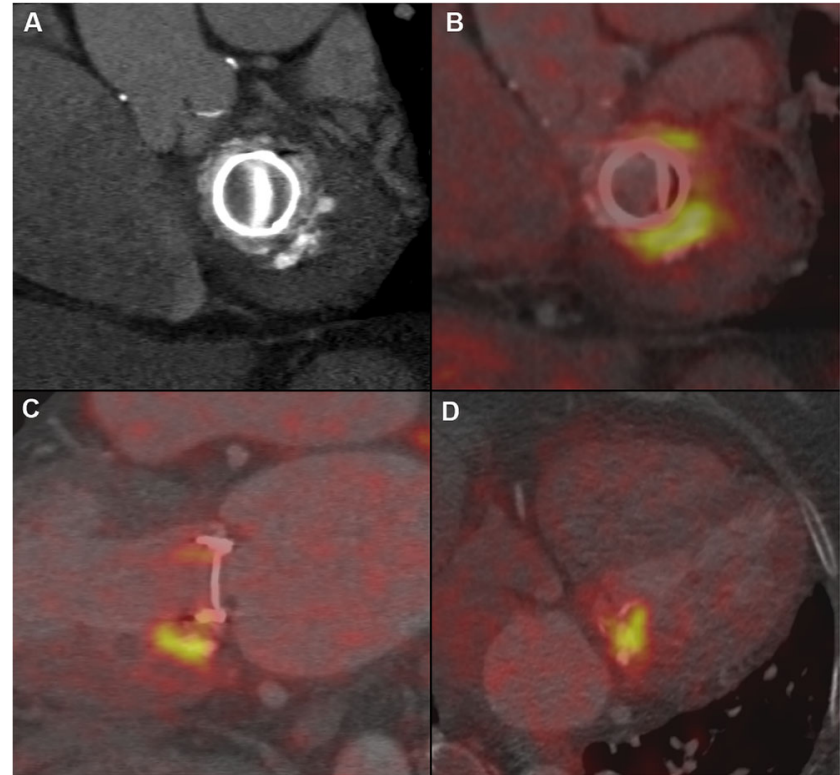

resected. The antibiotic regimen was repeated and the blood cultures remained negative after 3 months of follow-up.

\section{References}

1. Pizzi MN, Roque A, Fernández-Hidalgo N, Cuéllar-Calabria H, Ferreira-González I, Gonzàlez-Alujas MT, et al. Improving the diagnosis of infective endocarditis in prosthetic valves and intracardiac devices with 18F-fluordeoxyglucose positron emission tomography/ computed tomography angiography: initial results at an infective endocarditis referral center. Circulation. 2015;115:1113-26.

2. Saby L, Laas O, Habib G, Cammilleri S, Mancini J, Tessonnier L, et al. Positron emission tomography/computed tomography for diagnosis of prosthetic valve endocarditis: increased valvular $18 \mathrm{~F}-$ fluorodeoxyglucose uptake as a novel major criterion. J Am Coll Cardiol. 2013;61:2374-82. 